\title{
Latest results from T2K
}

\section{Jennifer Teresa Haigh, for the T2K Collaboration}

University of Warwick, Gibbet Hill Road, Coventry, UK

E-mail: j.haigh.2@warwick.ac.uk

The T2K experiment is a long-baseline neutrino oscillation project in Japan, consisting of an accelerator and near site at the J-PARC laboratory, and a far detector in the Kamioka mine. Results based on $1.5 \times 10^{21}$ protons on target are presented, with precise determinations of the parameters $\theta_{23}$ and $\Delta m_{32}^{2}$, and a measurement of $\theta_{13}$ complementary to reactor-based measurements. Preliminary indications of the neutrino mass hierarchy and CP violation are shown, and the future of the project is also discussed.

The 15th International Conference on Flavor Physics \& CP Violation 5-9 June 2017

Prague, Czech Republic 


\section{Introduction}

It is now well known[1] that the three neutrinos of the Standard Model possess non-zero mass, and that the three mass eigenstates $v_{i}(i=1,2,3)$ are not identical with the flavour eigenstates $v_{\alpha}(\alpha=e, \mu, \tau)$, but are related by a unitary transformation represented by the PMNS matrix $U_{\text {PMNS }}$, analogous to the CKM matrix for quark-sector mixing. This matrix is commonly parameterised using three mixing angles $\left(\theta_{12}, \theta_{13}, \theta_{23}\right)$, and a CP phase $\delta_{\mathrm{CP}}$. If all mixing angles are non-zero, and $\delta_{\mathrm{CP}} \notin(0, \pi)$, neutrino mixing will violate $\mathrm{CP}$ symmetry.

Mixing in the neutrino system leads to the phenomenon of neutrino oscillations - a neutrino produced in a known flavour state is in a superposition of mass states, whose relative phases will shift during propagation of the neutrino, leading to a finite probability that it will be observed in a different flavour state when detected later. Oscillation probabilities depend on the splittings between the squared mass values of the eigenstates rather than the absolute values. These are parameterised here as $\Delta m_{21}^{2}=m_{2}^{2}-m_{1}^{2}, \Delta m_{32}^{2}=\left|m_{3}^{2}-m_{2}^{2}\right|$. The ordering of the second and third mass states is unknown; here we use $\Delta m_{32}^{2}$ to refer to the modulus of the splitting. We refer to the case that $m_{3}>m_{2}$ as the Normal Hierarchy (NH), and $m_{3}<m_{2}$ as the Inverted Hierarchy (IH).

The values of the three mixing angles and the mass splittings have now been measured rather precisely. The key remaining unknowns accessible to oscillation measurements are:

- Whether CP symmetry is broken in the lepton sector, and the value of $\delta_{\mathrm{CP}}$.

- Whether the mass hierarchy is normal or inverted.

- Whether the mixing angle $\theta_{23}$ is greater or less than $45^{\circ}$ (the octant), or equal to $45^{\circ}$.

\section{The T2K experiment}

$\mathrm{T} 2 \mathrm{~K}[2]$ is a long baseline oscillation experiment based in Japan. $30 \mathrm{GeV}$ protons from the Main Ring synchrotron at the J-PARC facility are collided with a target to produce pions (plus a small number of other hadrons). These decay to produce neutrinos, mainly $v_{\mu}$, which are then observed by a suite of near detectors $280 \mathrm{~m}$ downstream of the target, and by a far detector $295 \mathrm{~km}$ away, in Kamioka mine. The hadrons are focused by magnetic horns after production, and by reversing the horn current, either positive or negative particles can be focused, leading to a beam dominated by $v_{\mu}$ or $\bar{v}_{\mu}$ respectively. Notably, both the main near detector, ND280, and the far detector, are positioned at $2.5^{\circ}$ from the beam axis, leading to a neutrino flux with energy narrowly focused around the point of maximum oscillation probability at around $600 \mathrm{MeV}$.

The far detector for T2K is the Super-Kamiokande water Cherenkov detector, located underground with $1 \mathrm{~km}$ rock overburden. This consists of a $50 \mathrm{kt}$ cylinder of water ( $22.5 \mathrm{kt}$ fiducial), separated into an inner detector and outer veto region, both instrumented with photomultiplier tubes. Charged leptons resulting from charged-current interactions of neutrinos with nuclei are detected via the Cherenkov radiation emitted as they traverse the detector volume. The particle energy is measured from the opening angle of the Cherenkov ring, and muons can be distinguished from electrons since they do not undergo significant scattering in the detector and thus produce cleaner rings. 
The main near detector, ND280, contains several subsystems, but the selections used for the oscillation analysis come from the tracker region. This consists of two Finely Grained Detectors (FGDs) made up of plastic scintillator bars, which act as the neutrino targets. The first, FGD1, is fully active, while FGD2 also contains water elements to enable the partial cancellation of systematics at the far detector, where the target is water. The FGDs are sandwiched between three Time Projection Chambers (TPCs), which provide particle identification information via particle $\frac{\mathrm{d} E}{\mathrm{~d} x}$. The detector is surrounded by a magnet providing a $0.4 \mathrm{~T}$ field, and it is therefore possible to measure particle momenta using curvature in the TPCs. In addition to the ND280, there is an onaxis beam monitor, INGRID, which measures the beam centre position and spread. It consists of 7 vertical and 7 horizontal modules in a plus configuration, with two additional diagonal modules. Each module is a $1 \times 1 \times 1 \mathrm{~m}^{3}$ iron-scintillator sampling calorimeter.

The primary measurements made by $\mathrm{T} 2 \mathrm{~K}$ are the probabilities, as a function of energy, for the $v_{\mu}$ to remain as a $v_{\mu}$, and to oscillate to a $v_{e}$, between production and detection at the far detector. To first order, the survival probability $P\left(v_{\mu} \rightarrow v_{\mu}\right)$ of a neutrino of energy $E_{v}$ is given by

$$
P\left(v_{\mu} \rightarrow v_{\mu}\right)=1-\sin ^{2}\left(2 \theta_{23}\right) \sin ^{2}\left(\frac{\Delta m_{32}^{2} L}{4 E_{v}}\right)
$$

where $L$ is the neutrino propagation distance. Making this measurement therefore allows to measure the mixing angle $\theta_{23}$ and the mass splitting $\Delta m_{32}^{2}$. Assuming CPT conservation, this survival probability should be the same for $v_{\mu}$ and $\bar{v}_{\mu}$.

The probability for a $\widehat{v}_{\mu}$ to oscillate to a $\widehat{V}_{e}$ is given approximately by

$$
\begin{aligned}
P\left(\bar{v}_{\mu} \rightarrow \breve{v}_{e}\right)= & 4 c_{13}^{2} s_{13}^{2} s_{23}^{2} \sin ^{2} \Delta_{31}\left(1+\frac{ \pm 2 a}{\Delta m_{31}^{2}}\left(1-2 s_{13}^{2}\right)\right) \\
& \mp 8 c_{13}^{2} c_{12} c_{23} s_{12} s_{13} s_{23} \sin \left(\delta_{\mathrm{CP}}\right) \sin \Delta_{32} \sin \Delta_{31} \sin \Delta_{21}
\end{aligned}
$$

$$
\text { where } c_{i j}=\cos \theta_{i j}, s_{i j}=\sin \theta_{i j}, \Delta_{i j}=\Delta m_{i j}^{2} \frac{L}{4 E_{v}}
$$

and non-leading terms producing identical effects for $v$ and $\bar{v}$ have been omitted, as has a small correction to matter effects. Here $a$ is a constant proportional to matter density along the neutrino path, whose sign is positive in the NH case and negative for the IH. It can be seen that both matter effects and $\mathrm{CP}$ violation produce asymmetry in oscillation probabilities between neutrinos and antineutrinos. This can lead to degeneracy between $\mathrm{CP}$ and hierarchy measurements; however at the $\mathrm{T} 2 \mathrm{~K}$ baseline, the asymmetry caused by maximal $\mathrm{CP}$ violation is roughly 2.5 times as large as matter effects.

The T2K experiment has been taking data since 2010, and since 2014 has been accumulating data in $\bar{v}$ mode as well as $v$ mode. The analysis presented here uses data from T2K Runs 1-7, corresponding to $0.75 \times 10^{21}$ protons incident on the T2K target in each mode, or around 250 selected events in the far detector. During the most recent run, Run 8 , the total collected sample in $v$ mode has been doubled. 


\section{Analysis}

The T2K oscillation analysis is based on a single fit of all the parameters $\left(\Delta m_{32}^{2}, \theta_{13}, \theta_{23}\right.$, $\delta_{\mathrm{CP}}$ ), to both $v_{\mu}$ and $v_{\mathrm{e}}$ datasets, in both $v$ and $\bar{v}$ running modes. External constraints on neutrino interaction models and solar oscillation parameters are used as priors in the fit. Hadron production in the target is simulated using a model based on results from the NA61 fixed-target experiment at CERN[3], and data from the INGRID on-axis detector is also used to constrain the flux model.

Three different fitters are used for the purposes of cross-checking results. Two of these are based on a "two-step" fit, where ND280 data is first used to constrain flux and cross-section uncertainties, and these constraints are then applied to a second fit to the Super-K datasets. The third method performs a simultaneous fit to data from ND280 and Super-K. Results from all three fitters are essentially identical.

\subsection{Constraints from ND280}

Data from $v_{\mu}$ interactions in the ND280 FGDs are used to constrain neutrino cross section parameters, as well as the expected beam flux at Super-K. Charged-current (CC) interactions $v_{l}+X \rightarrow l+X^{\prime}$, in both of the FGDs, are used. Events are split into samples based on the pion content of the final state; this splitting allows to constrain detailed parameters of the Monte Carlo model used for neutrino interactions. In particular, the most common topology, and also the main sample at Super-K, contains no pions in the final state; modulo corrections from intra-nuclear interactions and collective effects, this state essentially corresponds to Charged-current Quasielastic scattering (CCQE), where the neutrino interacts elastically with a nucleon, $v_{l}+N \rightarrow l+N^{\prime}$, leaving only a charged lepton, nucleon and recoil nucleus.

For neutrino mode running, events are divided into $\mathrm{CC} 0 \pi, \mathrm{CC} 1 \pi^{+}$and $\mathrm{CC}$-Other samples, where the $\mathrm{CC} 0 \pi$ sample is dominated by quasielastic events. For antineutrino running, there are separate samples for $v_{\mu}$ and $\bar{v}_{\mu}$, since there is significant $v_{\mu}$ contamination in the beam. In this case, the samples are divided up into CC-1Track (mainly quasielastic) and CC-NTrack. A subset of the ND280 samples is shown in Figure 1.

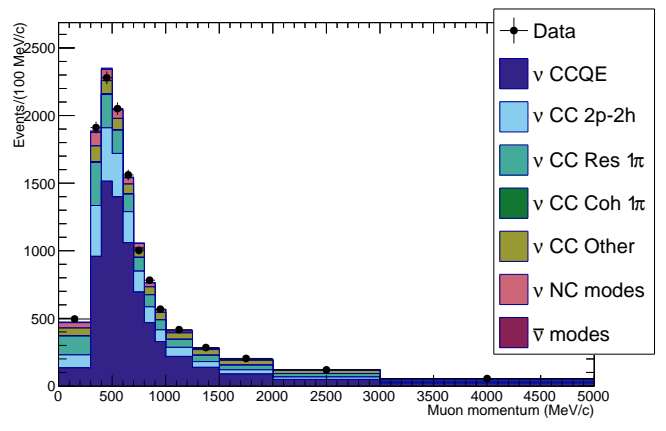

(a)

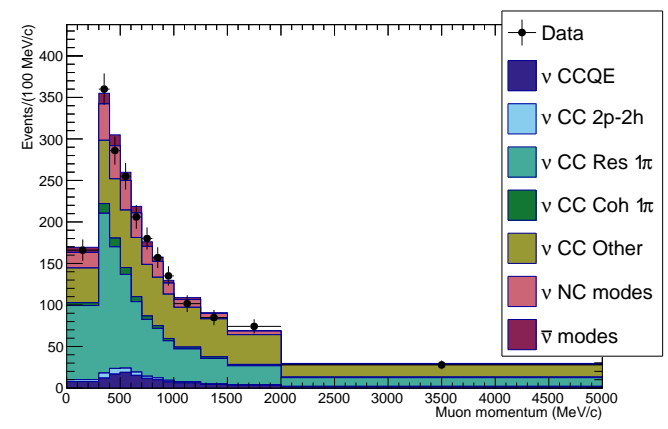

(b)

Figure 1: Examples of ND280 data samples, with the data accompanied by post-fit Monte Carlo distributions. Both samples are for events in FGD1, in neutrino beam mode - the left plot shows $\mathrm{CC} 0 \pi$-like events, and the right shows $\mathrm{CC} 1 \pi$. 
The effect on cross-section and flux uncertainties of fitting to the near detector data can be seen in Figure 2. In neutrino mode, the total systematic uncertainty on the Super-K single-ring event count is reduced from $12.1 \%$ to $4.9 \%$ for $v_{\mu}$ and $11.9 \%$ to $5.2 \%$ for $v_{\mathrm{e}}$, where the post-fit uncertainties arising from the parameters fitted to the near detector data and shown in the Figure are $2.5 \%$ and $2.7 \%$ respectively. The errors in $\bar{v}$ mode are similar, but errors relating to these samples are anyhow dominated by statistical effects.

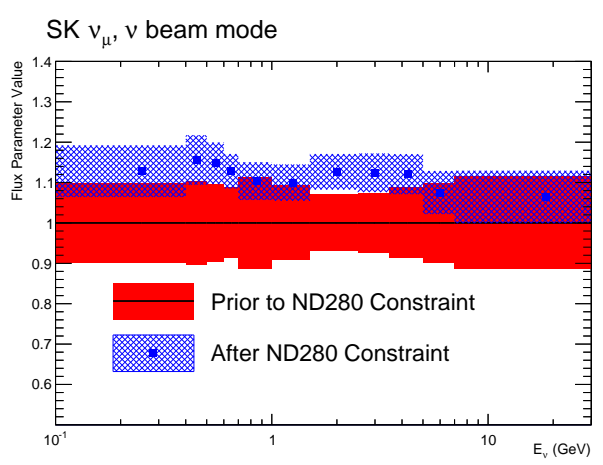

(a)

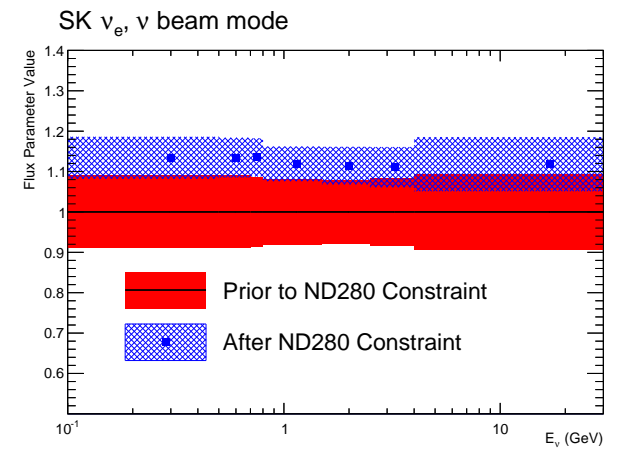

(b)

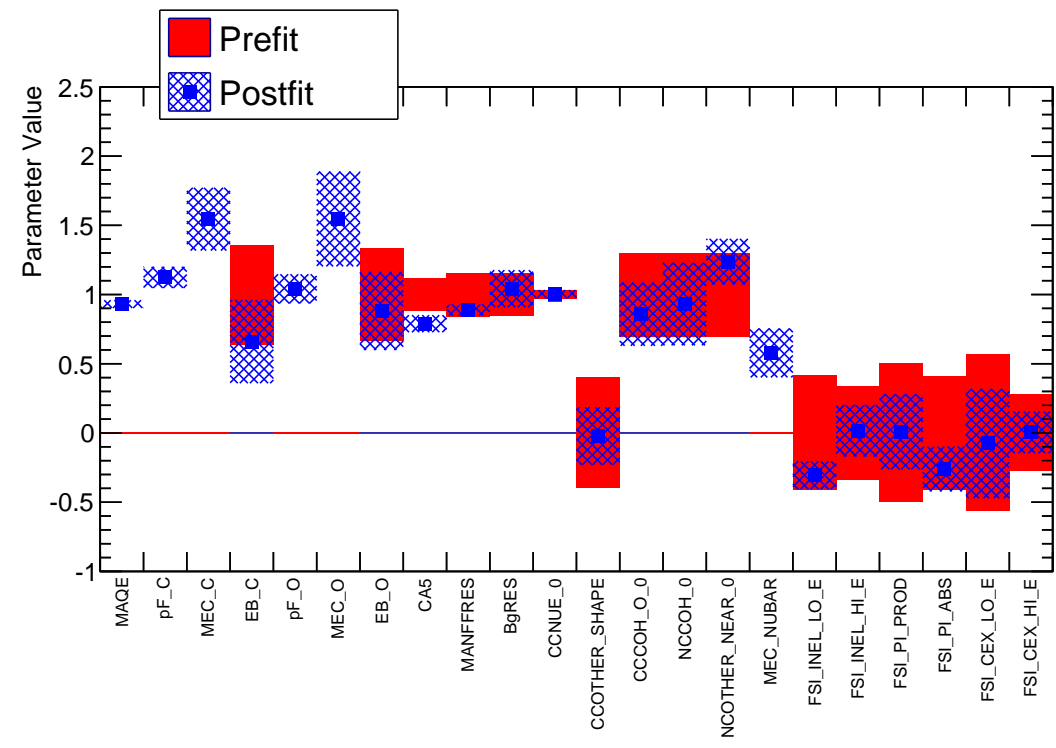

(c)

Figure 2: Neutrino-mode systematic errors before and after constraints from ND280 data. (a) shows errors on the $v_{\mu}$ flux, (b) shows errors on the $v_{e}$ flux, and (c) shows errors on cross-section parameters. In (a) and (b), the parameters are scaling factors for the flux in each bin relative to the nominal value.

\subsection{Super-K samples}

The primary data samples at Super-K comprise events containing a single Cherenkov ring, identified from ring shape as either muon or electron. These events arise predominantly from 
charged-current quasielastic neutrino interactions. In addition, for the neutrino-mode beam, a sample of events identified as charged-current interactions with an electron and charged pion in the final state is used to enhance the $v_{\mathrm{e}}$ statistics. These samples, along with the simulated distributions at the oscillation best fit point, and for the no-oscillation hypothesis, are shown in Figure 3.

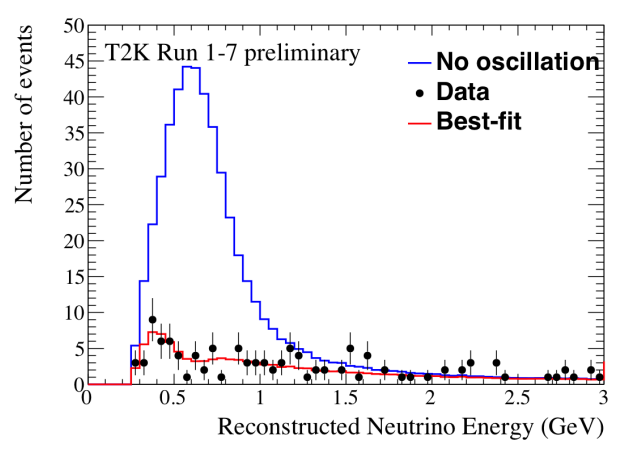

(a) $v$-mode 1 -Ring $\mu$-like

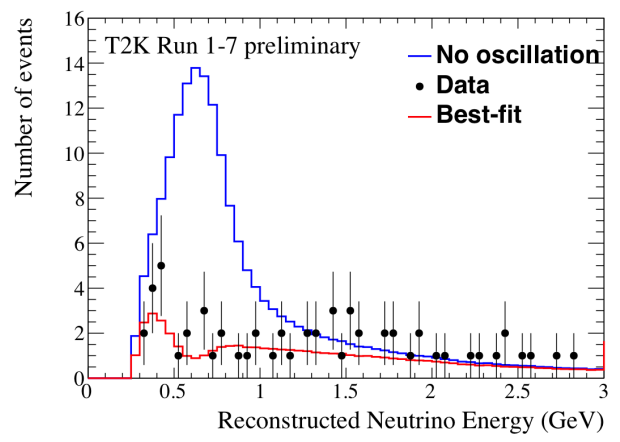

(c) $\bar{v}$-mode 1 -Ring $\mu$-like

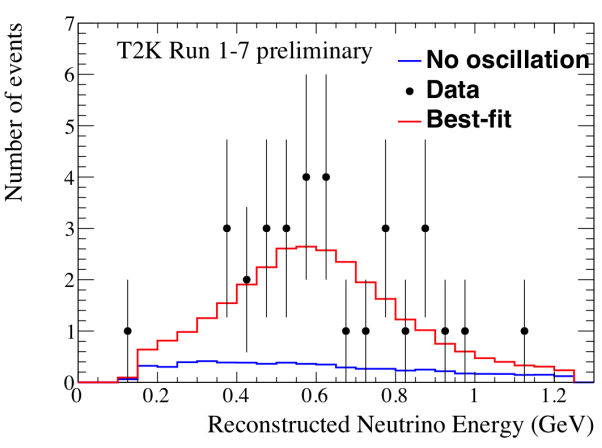

(b) $v$-mode 1-Ring e-like

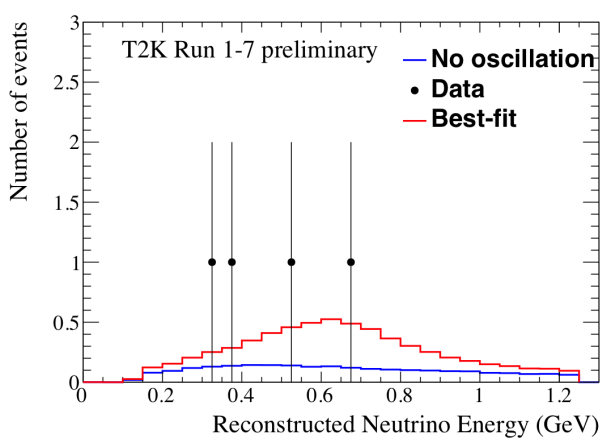

(d) $\bar{v}$-mode 1-Ring e-like

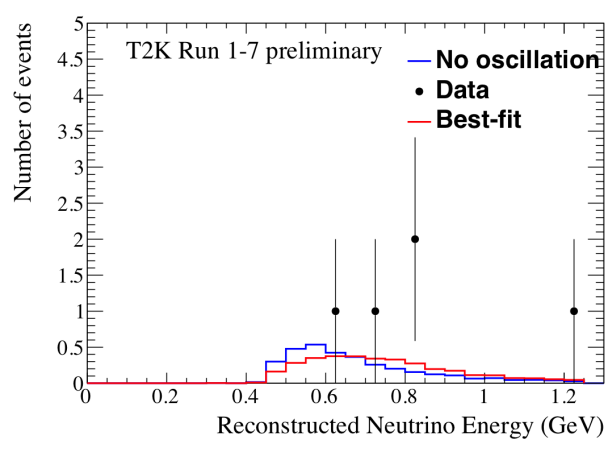

(e) $v$-mode CC1 $\pi$-like

Figure 3: Super-K data samples used in the oscillation analysis, shown with the prediction at the best fit point and for the no oscillation hypothesis.

A simultaneous fit of mixing parameters is made to all samples. Flat priors are used for the parameters to which $\mathrm{T} 2 \mathrm{~K}$ is sensitive, and Gaussian priors using external data as constraints are used for the solar mixing parameters. A second fit, using constraints[1] from reactor measurements for $\sin ^{2} \theta_{13}$, is also performed. For both fits, "nuisance" systematic parameters are dealt with by marginalisation, i.e. integration of the probability distribution. 


\section{Oscillation fit results}

The allowed region in the $\left(\Delta m_{32}^{2}, \sin ^{2} \theta_{23}\right)$ plane, for the $\mathrm{NH}$ only, along with the log-likelihood curves in $\sin ^{2} \theta_{23}$ for both hierarchies, are shown in Figure $4 \mathrm{a}$. No determination of the $\theta_{23}$ octant is possible with the current data. Figure $4 \mathrm{~b}$ shows allowed regions calculated using only $v$ or only $\bar{v}$ data - these are in excellent agreement and fully compatible with CPT conservation.

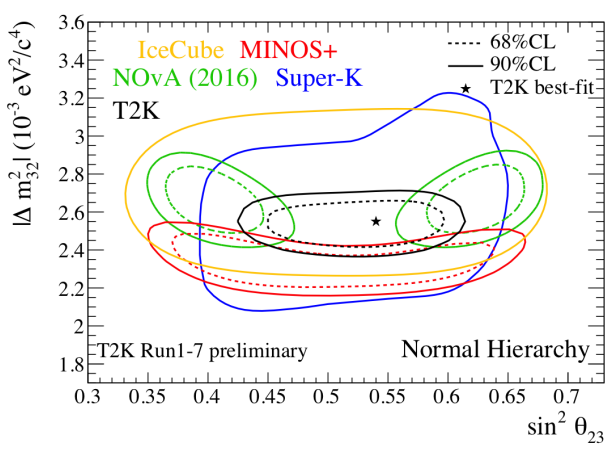

(a)

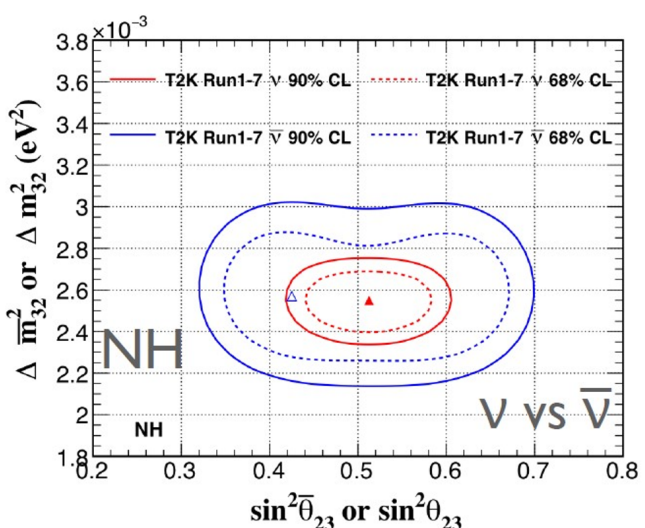

(b)

Figure 4: (a): T2K constraints on $\left(\Delta m_{32}^{2}, \sin ^{2} \theta_{23}\right)$ for the $\mathrm{NH}$, accompanied by the results from NOvA[5], Super-K[7], MINOS+[6] and IceCube[4]. (b): Constraints found using only $v$ or only $\bar{v}$ data.

The allowed regions in the $\left(\sin ^{2} \theta_{13}, \delta_{\mathrm{CP}}\right)$ plane are shown in Figure 5a, for both cases of the mass hierarchy. It can be seen that $\mathrm{T} 2 \mathrm{~K}$ is compatible with the value of $\sin ^{2} \theta_{13}$ taken from the Particle Data Group, which is driven by reactor measurements insensitive to $\delta_{\mathrm{CP}}$. Figure $5 \mathrm{~b}$ shows log-likelihood curves as a function of $\delta_{\mathrm{CP}}$ for both mass hierarchies, with the PDG mixing angle constraint applied. The normal hierarchy is weakly favoured. CP conservation is excluded at $90 \%$ C.L., and $\delta_{\mathrm{CP}}=0$ is excluded at $2 \sigma$ C.L..

\section{Future prospects}

The T2K experiment is approved to accumulate statistics of $7.8 \times 10^{21}$ protons on target roughly five times those used for the results presented here. This is forecast to be achieved by 2021, facilitated by improvements to the beamline at J-PARC, including an upgrade to the accelerator power supplies. In addition, a proposal has been submitted to the J-PARC PAC for an additional phase of the experiment. This T2K-II proposal includes statistics of $20 \times 10^{21}$ protons on target, as well as an upgraded near detector with improved acceptance for particles produced at a large angle with respect to the beam axis. The proposal assumes that a one-third reduction in systematic errors relative to the current analysis is achievable.

Figure 6 shows the expected sensitivity for measurement of $\left(\Delta m_{32}^{2}, \sin ^{2} \theta_{23}\right)$, for the full T2K run and for T2K-II. It can be seen that additional running will allow considerable improvement in the constraints on these parameters. Figure 7 shows the expected exclusion level for CP conservation, for both mass hierarchies, in the case that the true mass hierarchy is already known. It is not 


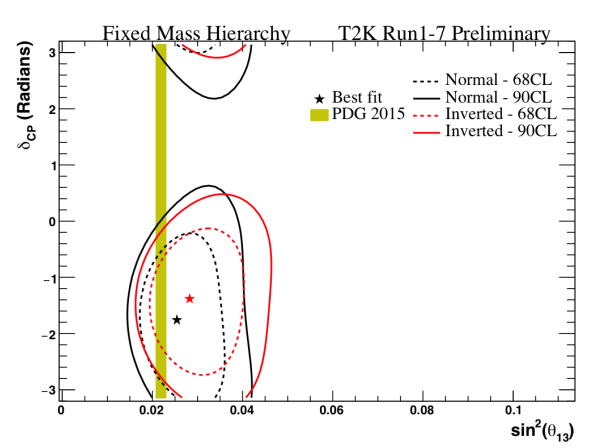

(a)

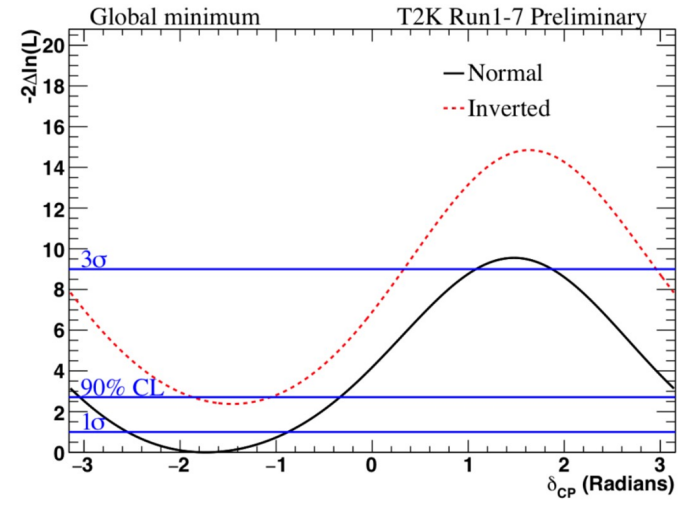

(b)

Figure 5: (a): T2K results for $\left(\sin ^{2} \theta_{13}, \delta_{\mathrm{CP}}\right)$, with the reactor constraint on the mixing angle shown as a coloured band. (b): The delta log-likelihood curves as a function of $\delta_{\mathrm{CP}}$ for both mass hierarchies, with the reactor constraint applied. The y axis shows the increase in the fit loglikelihood (actually $-2 \Delta \ln L$ ) relative to the best-fit point, which lies in the Normal Hierarchy, and the blue lines indicate the values of this parameter which correspond to given confidence levels to exclude a value.

expected that data from the initial $\mathrm{T} 2 \mathrm{~K}$ run will enable significant exclusion of $\mathrm{CP}$ conservation, regardless of the true value of $\delta_{\mathrm{CP}}$; however if $\mathrm{CP}$ violation is near-maximal, exclusion of $\mathrm{CP}$ conservation at $3 \sigma$ is achievable by T2K-II for the $\mathrm{NH}$, and also for the IH dependent on the sign of $\delta_{\mathrm{CP}}$.

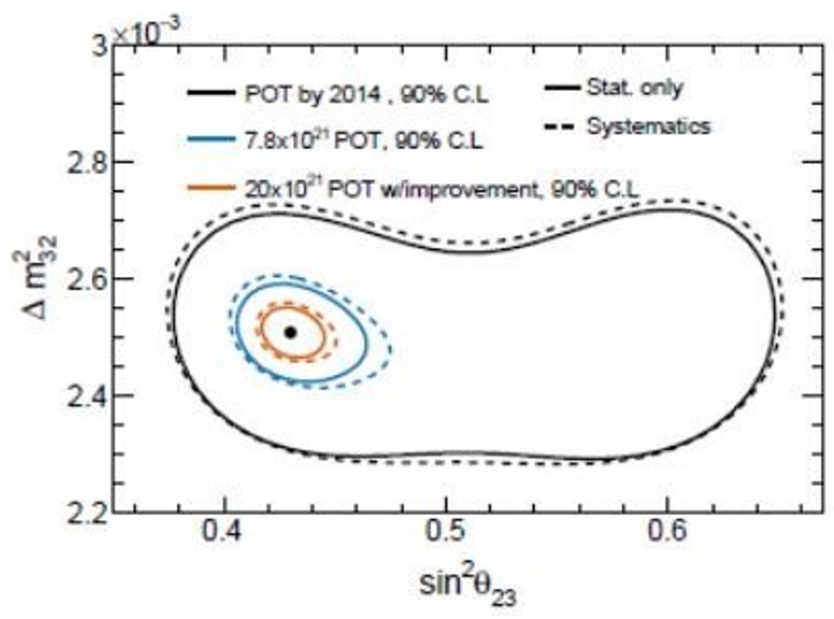

Figure 6: Predicted sensitivity to $\left(\Delta m_{32}^{2}, \sin ^{2} \theta_{23}\right)$ after the full T2K run, and for the T2K-II proposal, for the true value indicated in the plot.

\section{References}

[1] C. Patrignani et al. (Particle Data Group), Review of Particle Physics, Chin. Phys. C, 40, 100001 (2016). See section Neutrino mass, mixing and oscillations. 


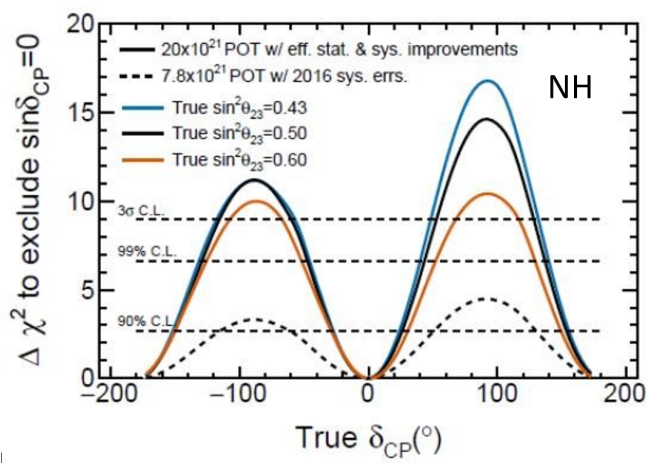

(a)

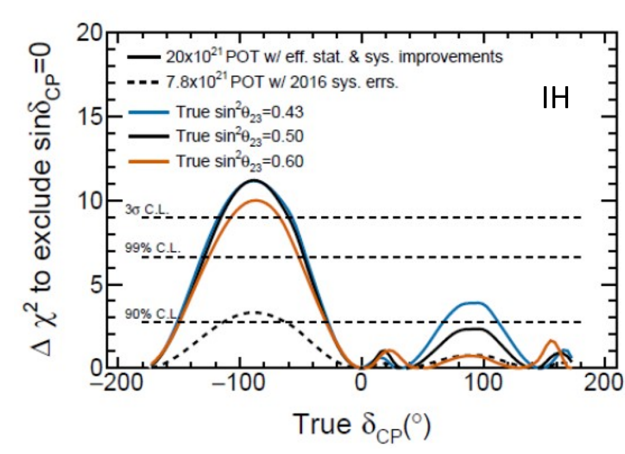

(b)

Figure 7: Predicted confidence for exclusion of CP conservation as a function of true $\delta_{\mathrm{CP}}$, for the $\mathrm{NH}(\mathrm{a})$, and the IH (b). Curves are shown for a range of reasonable values of $\sin ^{2} \theta_{23}$. Sensitivity is shown for the full $\mathrm{T} 2 \mathrm{~K}$ run and for T2K-II.

[2] K. Abe et al. (T2K Collaboration), The T2K Experiment, Nucl. Instrum. Meth. A 659 (2011) 106.

[3] N. Abgrall et al. (NA61/SHINE Collaboration), Measurements of $\pi^{ \pm}, K^{ \pm}, K_{S}^{o}, \Lambda$ and proton production in proton-carbon interactions at $31 \mathrm{GeV} / \mathrm{c}$ with the NA61/SHINE spectrometer at the CERN SPS, Eur. Phys. J. C 76 (2016) no.2, 84.

[4] M. G. Aartsen et al. (IceCube Collaboration), Determining neutrino oscillation parameters from atmospheric muon neutrino disappearance with three years of IceCube DeepCore data, Phys. Rev. D 91 (2015) no.7, 072004.

[5] P. Adamson et al. (NOvA Collaboration), Measurement of the Neutrino Mixing Angle $\theta_{23}$ in NOvA, Phys. Rev. Lett. 118 (2017) 151802.

[6] A. B. Sousa (MINOS and MINOS+ Collaborations), First MINOS+ data and new results from MINOS, in proceedings of Neutrino 2014, AIP Conf. Proc. 1666 (2015) 110004.

[7] R. Wendell (Super-Kamiokande Collaboration), Atmospheric Neutrino Oscillations at Super-Kamiokande, in proceedings of ICRC 2015, POS ( ICRC2015) 1062 (2015). 